nephron

Practice
Nephron 2017;137:264-267

DOI: $10.1159 / 000474960$
Received: March 1, 2017

Accepted after revision: April 2, 2017 Published online: June 9, 2017

\title{
Fibrotic Changes Mediating Acute Kidney Injury to Chronic Kidney Disease Transition
}

\author{
Eoghainín Ó hAinmhire Benjamin D. Humphreys \\ Division of Nephrology, Washington University School of Medicine, St. Louis, MO, USA
}

\section{Keywords}

Acute renal failure · Fibrosis · Ischemia-reperfusion injury

\begin{abstract}
End-stage renal disease (ESRD) is common, costly, and it results from progressive chronic kidney disease (CKD). ESRD claims many lives every year. It is increasingly recognized that episodes of acute kidney injury (AKI) predispose to the future development of CKD and ESRD. While our understanding of the pathophysiology of the AKI to CKD transition is improving, there are no validated therapeutic strategies to prevent this transition. In this review, we summarize the recent progress made in defining the cellular and molecular events underlying the AKI to CKD transition and highlight potential therapeutic targets and strategies to reduce the incidence of CKD following AKI.

(c) 2017 S. Karger AG, Basel
\end{abstract}

\section{Introduction}

Acute kidney injury (AKI) and chronic kidney disease (CKD) are both precursors to end-stage renal disease (ESRD), which led to over 46,000 deaths in 2013 [1]. While some patients with AKI make an apparent full re-

\section{KARGER}

(๑) 2017 S. Karger AG, Basel

E-Mail karger@karger.com

www.karger.com/nef covery, many others, perhaps even the majority, develop CKD in the future and are at an increased risk of developing ESRD. The pathophysiologic mechanisms that explain how an episode of AKI can lead to CKD and risk of ESRD are subjects of intense investigation. Understanding the cellular and molecular mechanisms underlying progression from $\mathrm{AKI}$ to $\mathrm{CKD}$ is required in order to develop novel therapeutics and diagnostic tools to treat this "silent killer."

\section{Tubular Injury and Repair}

AKI is characterized by a variety of cellular and structural injuries (online suppl. Fig. 1; for all online suppl. material, see www.karger.com/doi/10.1159/000474960) [2]. The proximal tubule (PT) is particularly susceptible to injury due to high metabolic activity. PT injury leads to dedifferentiation, characterized by distinct morpho-

Contribution from the AKI and CRRT 2017 Symposium at the 22nd International Conference on Advances in Critical Care Nephrology, Manchester Grand Hyatt, San Diego, CA, USA, March 7-10, 2017. This symposium was supported in part by the NIDDK funded University of Alabama at Birmingham-University of California San Diego O'Brien Center for Acute Kidney Injury Research (P30 DK079337). 
logical changes including loss of brush border, decreased cell size, fewer mitochondria, and elongated shape. Dedifferentiated cells also express injury markers and developmental genes, such as Pax2, while their proliferative rate increases [3]. Kusaba et al. [4] demonstrated using genetic lineage analysis that injured PT cells expressing kidney injury marker-1 (Kim-1) undergo dedifferentiation and proliferation in order to repair injured tubules. Kim-1 is a phosphatidylserine receptor that allows surviving epithelia to clear the tubule lumen of apoptotic fragments [5]. PT cells may also undergo a partial epithelial-to-mesenchymal transition (EMT) including enhanced migratory capacity during repair as demonstrated by the expression of Vimentin and Snail2 $[4,6]$. In many cases, PT can be fully reconstituted without apparent long-term structural damage in a process of successful repair (online suppl. Fig. S1b). However, some patients will undergo maladaptive repair, in which most function is restored, but an underlying fraction of cells remains damaged. This failed repair contributes to ongoing fibrotic processes and progresses over time to CKD (online suppl. Fig. S1b, c).

The progression of $\mathrm{PT}$ cells through the various states of differentiation and repair is being defined at the molecular level. A characteristic indication of successful repair is the subsequent downregulation of Kim-1 during redifferentiation, along with other dedifferentiation markers, such as Pax2, and EMT markers Vimentin and Snail1 [6]. The Sry-box family transcription factor, Sox9, is also induced during injury [7]. Similar to Kim-1, the expression of Sox9 is low in uninjured kidneys, and shows a dramatic upregulation $24 \mathrm{~h}$ after ischemia reperfusion injury (IRI) in murine models [7]. These Sox9+ injured PT cells are actively proliferating, with half of Sox9+ cells co-expressing Kim-1 [7]. Indeed, Kang et al. [8] showed that Sox9+ cells contributed to the repair of proximal, distal, and loop of Henle segments after folic acid injury and IRI. Using the Sox9-driven Cre-ER ${ }^{2}$ model crossed with R26R-tdTomato, Kumar et al. [7] showed that PT cells that initiated de novo expression of Sox9 after injury contributed the most to repair, further implicating dedifferentiation as the predominant mechanism of epithelial repair. Additionally, both PT-specific deletion of Sox9 in repair, and global deletion of Kim-1 during injury, exacerbated injury and delayed repair [7-9].

Although the fraction of PT that fail to repair after $\mathrm{AKI}$ is unknown, after moderate to severe AKI, a minority of PT cells and segments are characterized by ongoing injury and dedifferentiation. These cells fail to downregulate Kim-1, and chronic Kim-1 expression promotes proinflammatory cytokine secretion, peritu- bular inflammation, and interstitial fibrosis [5, 10]. These cells will also undergo G2/M cell cycle arrest, leading to secretion of pro-fibrotic growth factors, such as TGF $\beta$ and CTGF [11]. As additional insults occur to the kidney, this population of Kim-1 + dedifferentiated cells will increase, leading to CKD due to loss of PT integrity and function.

\section{Tubulointerstitial Fibrosis and Signaling}

While tubular epithelial cell damage is a core component in AKI to CKD transition, other growth factor and cytokine signaling pathways play important roles [12]. Aberrant paracrine signaling from the hedgehog ( $\mathrm{Hh})$ signaling pathway between the tubular cells and the interstitium contributes to tubulointerstitial fibrosis and capillary rarefaction, for example (online suppl. Fig. S1a, b). Hh ligands, including Sonic hedgehog (Shh) and Indian hedgehog (Ihh), signal by binding to their transmembrane receptor patched (Ptch1) [13], which activates intracellular transcription factors called Gli proteins [14]. These transcription factors include Gli1, Gli2, and Gli3. Gli1 expression marks a population of MSC-like pericytes in the outer medulla and inner cortex. These cells express PDGFR $\beta$ and are located adjacent to blood vessels in a perivascular niche [15]. Evidence supports the expression of both Ihh and Shh by tubular epithelium in kidney, with Shh expression seen in the collecting duct (CD), and Ihh expressed in S3 segment of the PT [13]. While Ptch1 is expressed in many kidney cell types, enrichment of the receptor is seen in perivascular interstitial cells [13].

During homeostasis, $\mathrm{Gli1}^{+}$pericytes stabilize vascular networks within the kidney through close contacts with microvascular endothelial cells (online suppl. Fig. S1a). Ablation of these pericytes is sufficient to drive capillary rarefaction in which the density of capillaries is significantly reduced. Most strikingly, ablation of $\mathrm{Gli}^{+}$cells also induces transient PT injury [16]. Kramann et al. [16] showed that loss of $\mathrm{Gli1}^{+}$cells lead to increased Kim-1 expression in the PT cells 10 days after ablation, which normalized by day 21 . This transient increase of Kim-1 in PT cells is similar to the type of injury witnessed in AKI (online suppl. Fig. S2). Additionally, both mild and severe IRI mouse models show increased capillary rarefaction and Kim- 1 expression, suggesting that loss of $\mathrm{Gli}^{+}$pericyte function may lead to AKI-like physiology $[15,16]$. To address this question, Kramann et al. [16] showed that during acute injury, $\mathrm{Gli}^{+}$pericytes detach from endothe- 
lial cells, expand into the cortex, and give rise to aSMA expressing myofibroblasts (online suppl. Fig. S1b, c) [15]. These myofibroblasts secrete extracellular matrix proteins, such as collagens and fibronectin, which accumulate in the interstitium, leading to increased hypoxia, decreased renal function, and eventually CKD. These data suggest that $\mathrm{Glil}^{+}$pericytes are one of the main progenitor cells contributing to the AKI-CKD transition. Indeed, ablation of these $\mathrm{Gli1}^{+}$perictyes after unilateral ureteral obstruction (UUO) significantly decreased interstitial fibrosis [15]. Collectively these observations suggest that the Hh pathway is a promising target for anti-fibrotic therapies. In support of this, Gli2 knockout in Gli1 ${ }^{+}$pericytes ameliorated kidney fibrosis, as did treatment with the Gli inhibitor, GANT61 [16].

Canonical Wnt signaling has been implicated in the AKI-CKD transition as well (online suppl. Fig. S1b, c) $[17,18]$. Wnt ligands are present in the early mouse embryo, and play a critical in nephrogenesis. Once nephrogenesis is complete, Wnt ligand expression decreases, and canonical Wnt signaling is repressed. However, during injury, both the epithelial and interstitial cells demonstrate activated canonical Wnt signaling [17]. DiRocco et al. [19] showed that Wnt4 ligand expression is restricted to the papillary $\mathrm{CD}$ epithelial cells in adult kidney, and not expressed within the cortex or medullary CD. Interestingly, during fibrosis, medullary myofibroblasts upregulate the Wnt4 ligand [19]. In fact, exogenous Wnt4 induced myofibroblast differentiation in vitro implicating Wnt4 as a fibrotic-inducing ligand. However, the knockout of Wnt 4 before injury did not ameliorate fibrosis. This is consistent with the fact that the kidney expresses many different Wnt ligands, which may compensate for the absence of a single one. On the other hand, constitutive activation of the canonical Wnt pathway (through $\beta$-catenin stabilization) in kidney interstitial pericytes was sufficient to drive myofibroblast formation in uninjured kidneys [19].

Using a different approach, Maarouf et al. [20] revealed that PT-specific and inducible expression of Wnt 1 was sufficient to induce interstitial fibrosis in the absence of any other injury. Wnt1 ligand had previously been shown to be secreted by PT epithelial in vitro and it is increased in injured kidneys [20]. A remarkable observation from this experiment was the complete absence of leukocyte infiltration or inflammatory cytokine expression in this model. Wnt1 induced interstitial fibrosis through the activation of canonical Wnt signaling and myofibroblast proliferation [20]. Several other studies are in agreement with these results, for example, the use of antagonists of the Wnt pathway such as Klotho [21], DKK1 [22], sFRP4 [23], and the chemical inhibitor ICG-001 [18]. By contrast, several other studies have demonstrated a reparative effect of Wnt, specifically on the tubular epithelial cells. Complete ablation of $\beta$-catenin in tubule cells exacerbated AKI by increasing p 53 and Bax expression [24]. This dual role of canonical Wnt signaling poses a dilemma. In AKI, Wnt signaling is necessary to force the proliferation and repair of tubule epithelial cells, while chronic overexpression of Wnt induces interstitial fibrosis and myofibroblast proliferation, potentially leading to AKI-CKD transition (online suppl. Fig. S1b, c) [18].

Another development pathway reactivated in AKI$\mathrm{CKD}$ transition is the notch signaling pathway (online suppl. Fig. S1b, c). Notch signaling regulates various cellular processes depending on the cell type. In stem cells, notch is involved in differentiation, and is often overexpressed in many carcinomas $[25,26]$. Notch plays a crucial role in both podocyte and PT development in the kidney, with many different notch ligands and receptors being expressed throughout nephrogenesis [27]. The expression of notch diminishes after development, with very little signaling in the mature kidney [27]. However, during AKI, the notch pathway is reactivated in tubular epithelial cells [28]. After IRI, an upregulation of notch2, delta-1, and Hes-1 was seen in the PT epithelial cells [28]. Additionally, jagged-1 and Hes-1 were found to be upregulated in the tubule epithelial of diabetic nephropathy patients [29]. Mariana et al. [30] later showed that activated notch signaling was not unique to diabetic nephropathy, with notch-1 expression correlating with the degree of tubulointerstitial fibrosis in a wide variety of kidney diseases. In animal models, Djudjaj et al. [31] demonstrated that genetic deletion of notch-3 in UUO leads to a reduction in tubular injury, along with a decrease in collagen deposition. Finally, Bielesz et al. [32] found that inhibiting notch, both genetically and pharmacologically using $\gamma$-secretase inhibitor, reduced tubulointerstitial fibrosis in UUO and folic acid models. This data indicates that notch signaling is also an important target for therapeutic intervention (online suppl. Fig. S1c).

\section{Conclusion and Future Therapeutics}

Despite the cellular complexity of AKI and CKD, progress has been made in defining critical pathways that represent attractive therapeutic targets. While many drug 
candidates for the pathways listed above are in clinical trials for other indications, such as various cancers, few are being tested in kidney disease. While clinical trials in $\mathrm{AKI}$ and CKD pose a unique set of challenges, this growing list of candidate therapeutics to prevent the AKI to CKD transition suggests that a therapeutic breakthrough is possible in the next decade. Deciphering the balance between reparative vs. fibrotic signaling after acute injury should lead to novel therapeutics for patients experiencing kidney injury.

\section{Acknowledgments}

E.O. is supported by grant T32DK007126 from the NIDDK. Work in the Humphreys Laboratory is supported by grants DK103050, DK103740, DK107374, and DK104308 from the NIDDK and an Established Investigator Award from the American Heart Association.

\section{Disclosure Statement}

The authors has no conflict of interest to declare.

\section{References}

$1 \mathrm{Xu}$ J, Murphy SL, Kochanek KD, Bastian BA: Deaths: final data for 2013. Natl Vital Stat Rep 2016;64:1-119.

2 Ferenbach DA, Bonventre JV: Mechanisms of maladaptive repair after AKI leading to accelerated kidney ageing and CKD. Nat Rev Nephrol 2015;11:264-276.

3 Chang-Panesso M, Humphreys BD: Cellular plasticity in kidney injury and repair. Nat Rev Nephrol 2017;13:39-46.

4 Kusaba T, Lalli M, Kramann R, Kobayashi A, Humphreys BD: Differentiated kidney epithelial cells repair injured proximal tubule. Proc Natl Acad Sci U S A 2014;111:1527-1532.

5 Ichimura T, Asseldonk EJ, Humphreys BD, Gunaratnam L, Duffield JS, Bonventre JV: Kidney injury molecule- 1 is a phosphatidylserine receptor that confers a phagocytic phenotype on epithelial cells. J Clin Invest 2008; 118:1657-1668

6 Grande MT, Sanchez-Laorden B, Lopez-Blau C, De Frutos CA, Boutet A, Arevalo M, et al: Snaill-induced partial epithelial-to-mesenchymal transition drives renal fibrosis in mice and can be targeted to reverse established disease. Nat Med 2015;21:989-997.

7 Kumar S, Liu J, Pang P, Krautzberger AM, Reginensi A, Akiyama H, et al: Sox9 activation highlights a cellular pathway of renal repair in the acutely injured mammalian kidney. Cell Rep 2015;12:1325-1338.

8 Kang HM, Huang S, Reidy K, Han SH, Chinga F, Susztak K: Sox9-positive progenitor cells play a key role in renal tubule epithelial regeneration in mice. Cell Rep 2016;14:861-871.

9 Ismail OZ, Zhang X, Wei J, Haig A, Denker BM, Suri RS, et al: Kidney injury molecule-1 protects against Ga12 activation and tissue damage in renal ischemia-reperfusion injury. Am J Pathol 2015;185:1207-1215.

10 Humphreys BD, Xu F, Sabbisetti V, Grgic I, Movahedi Naini S, Wang N, et al: Chronic epithelial kidney injury molecule-1 expression causes murine kidney fibrosis. J Clin Invest 2013;123:4023-4035.

11 Yang L, Besschetnova TY, Brooks CR, Shah JV, Bonventre JV: Epithelial cell cycle arrest in
G2/M mediates kidney fibrosis after injury. Nat Med 2010;16:535-543, 1p following 143.

12 Venkatachalam MA, Weinberg JM, Kriz W, Bidani AK: Failed tubule recovery, AKI-CKD transition, and kidney disease progression. J Am Soc Nephrol 2015;26:1765-1776.

13 Fabian SL, Penchev RR, St-Jacques B, Rao AN, Sipilä P, West KA, et al: Hedgehog-Gli pathway activation during kidney fibrosis. Am J Pathol 2012;180:1441-1453.

14 Zhou D, Tan RJ, Liu Y: Sonic hedgehog signaling in kidney fibrosis: a master communicator. Sci China Life Sci 2016;59:920-929.

15 Kramann R, Schneider RK, DiRocco DP, Machado F, Fleig S, Bondzie PA, et al: Perivascular Gli1+ progenitors are key contributors to injury-induced organ fibrosis. Cell Stem Cell 2015;16:51-66.

16 Kramann R, Fleig SV, Schneider RK, Fabian SL, DiRocco DP, Maarouf O, et al: Pharmacological GLI2 inhibition prevents myofibroblast cell-cycle progression and reduces kidney fibrosis. J Clin Invest 2015;125:2935-2951.

17 Maarouf OH, Ikeda Y, Humphreys BD: Wnt signaling in kidney tubulointerstitium during disease. Histol Histopathol 2015;30:163-171.

18 Zhou D, Tan RJ, Fu H, Liu Y: Wnt/ $\beta$-catenin signaling in kidney injury and repair: a double-edged sword. Lab Invest 2016;96:156167.

19 DiRocco DP, Kobayashi A, Taketo MM, McMahon AP, Humphreys BD: Wnt $4 / \beta$-catenin signaling in medullary kidney myofibroblasts. J Am Soc Nephrol 2013;24:1399-1412.

20 Maarouf $\mathrm{OH}$, Aravamudhan A, Rangarajan D, Kusaba T, Zhang V, Welborn J, et al: Paracrine Wnt1 drives interstitial fibrosis without inflammation by tubulointerstitial cross-talk. J Am Soc Nephrol 2016;27:781-790.

21 Zhou L, Li Y, Zhou D, Tan RJ, Liu Y: Loss of Klotho contributes to kidney injury by derepression of $\mathrm{Wnt} / \beta$-catenin signaling. J Am Soc Nephrol 2013;24:771-785.

22 He W, Dai C, Li Y, Zeng G, Monga SP, Liu Y: Wnt/beta-catenin signaling promotes renal interstitial fibrosis. J Am Soc Nephrol 2009; 20:765-776.
23 Surendran K, Schiavi S, Hruska KA: Wnt-dependent beta-catenin signaling is activated after unilateral ureteral obstruction, and recombinant secreted frizzled-related protein 4 alters the progression of renal fibrosis. J Am Soc Nephrol 2005;16:2373-2384.

24 Zhou D, Li Y, Lin L, Zhou L, Igarashi P, Liu Y: Tubule-specific ablation of endogenous $\beta$-catenin aggravates acute kidney injury in mice. Kidney Int 2012;82:537-547.

25 Andersson ER, Sandberg R, Lendahl U: Notch signaling: simplicity in design, versatility in function. Development 2011;138: 3593-3612.

26 Yuan X, Wu H, Xu H, Xiong H, Chu Q, Yu S, et al: Notch signaling: an emerging therapeutic target for cancer treatment. Cancer Lett 2015;369:20-27.

27 Sharma S, Sirin Y, Susztak K: The story of Notch and chronic kidney disease. Curr Opin Nephrol Hypertens 2011;20:56-61.

28 Kobayashi T, Terada Y, Kuwana H, Tanaka H, Okado T, Kuwahara M, et al: Expression and function of the Delta-1/Notch-2/Hes-1 pathway during experimental acute kidney injury. Kidney Int 2008;73:1240-1250.

29 Walsh DW, Roxburgh SA, McGettigan P, Berthier CC, Higgins DG, Kretzler M, et al: Co-regulation of Gremlin and Notch signalling in diabetic nephropathy. Biochim Biophys Acta 2008;1782:10-21.

30 Murea M, Park JK, Sharma S, Kato H, Gruenwald A, Niranjan T, et al: Expression of notch pathway proteins correlates with albuminuria, glomerulosclerosis, and renal function. Kidney Int 2010;78:514-522.

31 Djudjaj S, Chatziantoniou C, Raffetseder U, Guerrot D, Dussaule JC, Boor P, et al: Notch-3 receptor activation drives inflammation and fibrosis following tubulointerstitial kidney injury. J Pathol 2012;228:286299.

32 Bielesz B, Sirin Y, Si H, Niranjan T, Gruenwald A, Ahn S, et al: Epithelial notch signaling regulates interstitial fibrosis development in the kidneys of mice and humans. J Clin Invest 2010;120:4040-4054. 